\title{
Rücktritt als Präsident des SPV und Aufruf für eine vernünftige Psychotherapiepolitik
}

\author{
Thomas Merki (Zürich)
}

In einer emotional geführten Debatte nahm die Mitgliederversammlung des Schweizer Psychotherapeutinnen und Psychotherapeuten Verbandes SPV am 15. März 2008 einen vom Präsidenten der Schweizer Charta für Psychotherapie eingereichten Antrag zur Änderung der Aufnahmebedingungen in den SPV gegen die Empfehlung des Vorstandes an. Somit sollen auch wieder AbsolventInnen anderer Studienrichtungen Mitglieder im SPV werden können. Zur Erinnerung: Vor Jahresfrist hat ebenfalls die Mitgliederversammlung ein abgeschlossenes Hochschulstudium in Psychologie oder Medizin an einer Schweizer Hochschule inklusive Psychopathologie als Voraussetzung für die Mitgliedschaft festgesetzt! Notabene dieselben Voraussetzungen, wie der Kanton Zürich für die Erlangung der Praxisbewilligung verlangt oder wie im Tarmed für die delegiert arbeitenden PsychotherapeutInnen verlangt wird.

\section{Warum die Beschränkung der Grundausbildung auf ein Psychologie- respektive Medizinstudium?}

Die Beschränkung auf ein Psychologie- oder Medizinstudium ist das Resultat einer langen politischen Auseinandersetzung um die Anerkennung des Berufes PsychotherapeutIn als Heilberuf und deren Integration in die Gesundheitsversorgung. In der Frage der Grundausbildung haben sich die Psychologenverbände mit den Universitäten und den Fachhochschulen im Rücken durchgesetzt. Dabei vertraten die Vertreter der psychotherapeutischen Berufsverbände richtigerweise ein Psychotherapie-Berufsbild, welches die Psychotherapie als einen eigenständigen, wissenschaftlichen Beruf versteht. Diese Sichtweise fusst auf einem in der Schweiz historisch gewachsenen Verständnis der Psychotherapie. Allerdings wurde in den letzten Jahren Psychotherapieforschung hauptsächlich von PsychologInnen betrieben, argwöhnisch beobachtet von den PsychotherapeutInnen. Damit einhergehend sank der Einfluss der psychotherapeutischen Methoden in den Universitätsinstituten und begünstigte die heutige Dominanz der einseitig verhaltenspsychologisch ausgerichteten Techniken.

Unbestritten bestehen heute bei den Gesundheitsberufen, wie bei anderen Berufen auch, eine viel höhere Regelungsdichte und ein höherer Regelungsbedarf 
als noch vor zwanzig oder dreissig Jahren. So bedauerlich uns die Einschränkung bei der Grundvoraussetzung zum PsychotherapeutInnenberuf scheinen mag, so dringend stellt sich uns die Aufgabe, sich dieser Entwicklung zu stellen. Dabei orientierte ich mich während meiner Präsidentschaft an den Kernaufgaben eines Berufsverbandes: Die Vertretung der Interessen der angeschlossenen PsychotherapeutInnen und eine starke Einflussnahme in der Frage der psychotherapeutischen Versorgung der Bevölkerung. Im derzeitigen nicht sehr Psychotherapie freundlichen politischen Umfeld gibt es starke Kräfte, die die Psychotherapie am liebsten abschaffen oder auf ein «psychiatrisches Minimum» zurückdrängen möchten. Die Auseinandersetzung mit diesen Entwicklungen ist vordringlich und wichtiger als das Festhalten an überkommenen, selbstreferentiellen Ausbildungskonzepten oder am nicht anerkannten und quer in der Bildungslandschaft stehenden Master-Studiengang, wie ihn die Charta anbietet.

Um den Einfluss der nicht-ärztlichen oder psychologischen Psychotherapie (oder wie auch immer wir uns nennen mögen) zu erhalten und zu verstärken, ist eine eidgenössische Regelung notwendig. Die Verzettelung auf mehr als zwei Dutzend verschiedene kantonale Regelungen bringt uns vor allem etwas: Es schwächt unsere Position nachhaltig! DerVorentwurf für ein Gesetz über die psychologischen Berufe (PsyG), bei dem alle namhaften Kräfte mitgearbeitet haben, bringt uns genau diese eidgenössische Regelung und verdient deshalb unsere Unterstützung. Auch wenn in diesem Entwurf die Psychotherapie in einem Atemzug wie die anderen psychologischen Berufe genannt wird.

\section{Was ist zu tun?}

Als Reaktion auf den eingangs geschilderten Mitgliederversammlungsbeschluss zog ich nach reiflicher Überlegung die Konsequenz und trat als Präsident des SPV vorzeitig zurück. Dies aus der Einsicht heraus, dass sich die Weiterführung des zähen Kampfes mit den einseitig bewahrenden Kräften innerhalb des SPV nicht weiter lohnt. Deshalb habe ich zusammen mit meinem Vorgänger Raimund Dörr im Mai den «Aufruf für eine vernünftige Psychotherapiepolitik!» lanciert. Ziel dieses Aufrufes ist es, diejenigen Kräfte zu sammeln, die ebenfalls der Meinung sind, dass

, die Psychotherapie als ein wichtiger Bestandteil in der Gesundheitspolitik verankert werden soll

, die Psychotherapie in einem Gesetz über die psychologischen Berufe (PsyG) geregelt werden soll 
, die PsychotherapeutInnen mittelfristig in den Leistungskatalog der Krankenpflege-Leistungsverordnung (KLV) aufgenommen werden sollen

, und in der Zwischenzeit die Verbesserung der Arbeitssituation von praktizierenden PsychotherapeutInnen (zum Beispiel Anhebung des Tarifs im neuen IV-Vertrag, Vertragsabschlüsse mit Krankenkassen und anderen Versicherungsträgern) angestrebt werden sollen.

Diese Ziele bedingen grösste Anstrengungen zur Qualitätssicherung in der Grund- und Weiterbildung. Aus diesem Grund haben wir uns für die Vereinheitlichung der Grundausbildung und für ein Grundstudium in Psychologie oder Medizin ausgesprochen. Ganz wichtig dabei ist aber, dass die bisherigen Übergangs- und altrechtlich erworbenen Qualifikationen und Bewilligungen erhalten bleiben!

Ob es zur Durchsetzung dieser Politik einen neuen Berufsverband braucht, wird sich weisen. Ein erstes Treffen der UnterzeichnerInnen des Aufrufs fand anfangs Juli in Zürich statt. Priorität hat die Bündelung der Kräfte und nicht die Bildung eines bürokratischen Apparates. Denkbar ist auch das Zusammengehen mit den fortschrittlichen, psychotherapeutischen Kräften innerhalb der Föderation der Schweizer PsychologInnen FSP und des Schweizerischen Berufsverbandes für angewandte Psychologie SBAP. 